\title{
CARACTERIZACIÓN DE MATERIALES PARA EL DISEÑO Y DESARROLLO DE COLECTORES Y RECEPTORES DE RADIACIÓN SOLAR TÉRMICA DE CONCENTRACIÓN
}

\section{CHARACTERIZATION OF MATERIALS FOR THE DESIGN AND DEVELOPMENT OF COLLECTORS AND RECEIVERS OF CONCENTRATION SOLAR THERMAL RADIATION}

\section{Doctorando:}

\section{Leonardo Andrés Corujo}

Facultad Regional La Plata, Universidad Tecnológica Nacional - Facultad de Ciencias Astronómicas y Geofísicas, Universidad Nacional de La Plata - Laboratorio de Óptica Calibraciones y Ensayos - Argentina.

leocorujo@gmail.com

\section{Director/a:}

Ana María Castro Luna

Co-director/a:

Luis César Martorelli 


\title{
Resumen
}

El trabajo que se presenta muestra el estudio de diferentes materiales a utilizar en el diseño de colectores y receptores para usar en la construcción de prototipos innovadores que usen tecnología basada en el empleo de la Energía Solar Térmica de Concentración (ESTC), para la generación de calor y electricidad.

Se estudiaron las propiedades ópticas de los diferentes materiales propuestos, así como su estabilidad térmica y durabilidad ya que con ellos se construirán, específicamente, los componentes de la tecnología de sistema de Colector cilíndrico Parabólico (CCP). Con el propósito de alcanzar mayor eficiencia en el proceso de transformación de energía, se optimizaron, a través del diseño y la simulación, diversas geometrías del dispositivo, que se articularán a los sistemas de seguimiento de la radiación solar en la unidad CCP prototipo, tomando en consideración las regiones geográficas de aplicación.

Palabras clave: Energía - Solar - Concentración - Receptores.

\begin{abstract}
The work presented shows the study of different materials to be used in the design of collectors and receivers to be used in the construction of innovative prototypes that use technology based on the use of Solar Thermal Concentration Energy (ESTC), for the generation of heat and electricity.

The optical properties of the different materials proposed were studied, as well as their thermal stability and durability, since with them the components of the Parabolic Cylindrical Collector (CCP) system technology will be specifically built. In order to achieve greater efficiency in the energy transformation process, various geometries of the device were optimized through design and simulation, which will be linked to the solar radiation monitoring systems in the prototype CCP unit, taking into consideration the geographical regions of application.
\end{abstract}

Keywords: Energy - Solar - Concentration - Receptors 


\section{Introducción}

Funcionalmente, los sistemas que aprovechan la radiación solar térmica ESTC resultan de la combinación de cuatro componentes principales:

i) El colector, que capta la radiación solar para convertirla en otro tipo de energía, siendo el sistema de incidencia/reflexión en superficies el más utilizado. Existen diferentes tecnologías de concentración de la energía solar tales como la del disco o cilindro parabólicos, la de torre central y la de reflexión lineal Fresnel (Gordon, 2013); (Luján, Aires, y 2004, n.d.).

ii) El receptor que recibe la radiación, y convierte la energía electromagnética del sol en térmica, utiliza materiales de alta relación de absortividad /emisividad. El concentrador y el receptor se mueven solidariamente, siguiendo continuamente al sol. (Gordon, 2013); (Luján, Aires, y 2004, n.d.).

iii) El almacenador que almacena el calor y mantiene en espera parte de la energía captada, modulando su entrada y salida. (Gordon, 2013); (Luján, Aires, y 2004, n.d.).

iv) La unidad de potencia donde se utiliza la energía térmica, ya sea directamente, como calor o transformada para generar electricidad. (Gordon, 2013); (Luján, Aires, y 2004, n.d.).

\section{DISEÑO Y DESARROLLO DE UN COLECTOR CILÍNDRICO PARABÓLICO (CCP)}

En función del tema de investigación de tesis propuesto; Caracterización de Materiales para el Diseño y Desarrollo de Colectores y Receptores de Radiación Solar Térmica de Concentración, y en el marco del proyecto de Innovación y Transferencia en Áreas Prioritarias (PIT-AP-BA) desde 2016. Se ha diseñado y desarrollado un CCP (Colector Cilíndrico Parabólico) para la producción de vapor, con uso potencial en diversos procesos, como es el caso de los secaderos solares de productos alimenticios, evaporación en (RSU) Residuos Sólidos Urbanos o la producción de electricidad usando además una caldera de biomasa. (Fernández-García, Zarza, Valenzuela, \& Pérez, 2010), (Matos, 2017); (L. Martorelli, Panaroni, Alvarez, Corujo, \& Cervini, 2020); (L. C. Martorelli, n.d.); (Mujica, Panaroni, \& Corujo, 2019); (Parabólico, n.d.); (Panaroni et al., 2017).

Se participo en la investigación y desarrollo del dispositivo, específicamente en la caracterización de materiales, el diseño de la estructura, y el diseño del colector y el receptor; y en la realización del prototipo del sistema. Figura 1, 2,3.

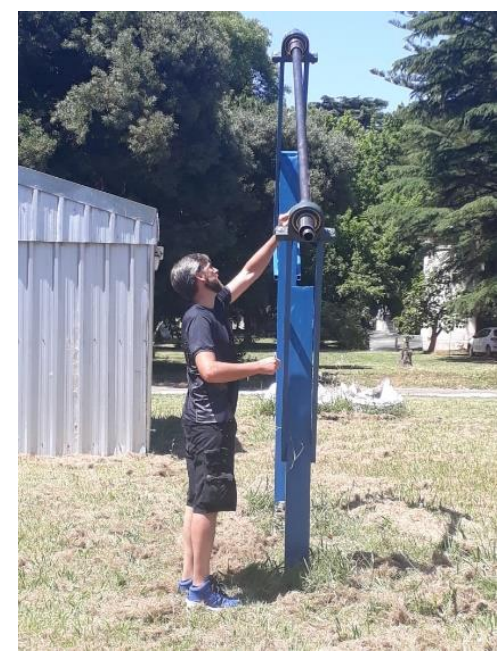

Figura 1. Vista frontal, receptor

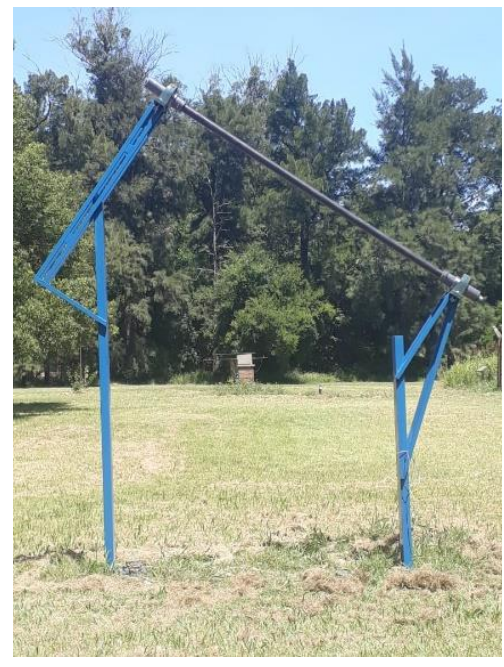

Figura 2. Vista lateral izquierda

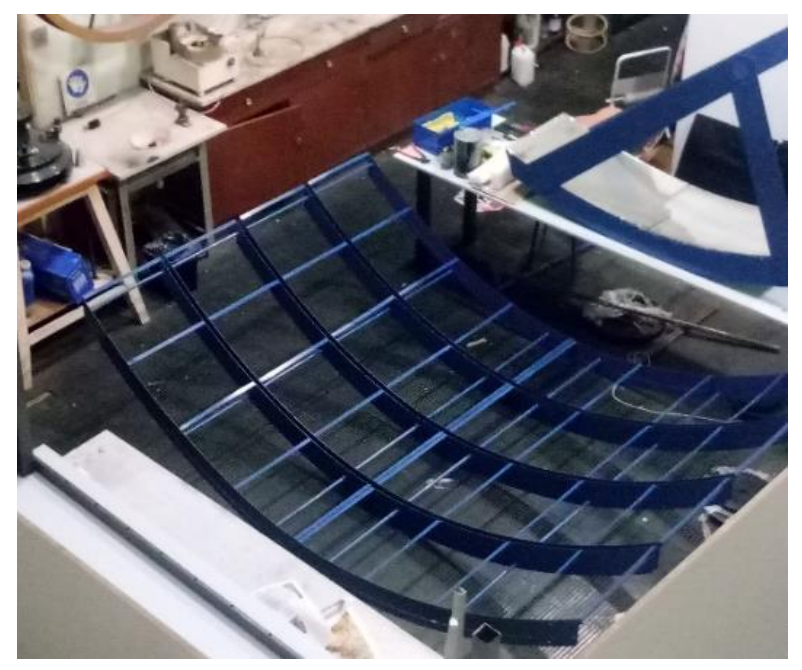

Figura 3. Estructura colectora 


\section{Desarrollo}

\section{Materiales para el receptor del colector $\mathrm{CCP}$}

Para el receptor se estudió y seleccionó caño de acero normalizado, ASTM A 53 Grado B, de conducción y sin costura, para altas presiones y temperaturas. Realizado por extrusión en caliente con sus aleaciones correspondientes y posteriores tratamientos térmicos que mejoran las propiedades mecánicas del acero (especialmente la dureza, la resistencia y la elasticidad) que se adecuen a las consideraciones de diseño del sistema CCP (Colector Cilíndrico Parabólico) y específicamente en el receptor; (Groover, 2007). Figuras 1 y 2 . Tabla 1.

\section{Composición química y mecánica de caño de acero ASTM A53 GRADO B}

\begin{tabular}{|c|c|c|c|c|c|c|c|c|c|c|c|c|}
\hline \multirow{3}{*}{$\begin{array}{c}\text { ASTM A } \\
53 \\
\text { GRADO }\end{array}$} & \multicolumn{9}{|c|}{$\begin{array}{l}\text { Composición } \\
\text { Química }\end{array}$} & \multicolumn{3}{|l|}{$\begin{array}{l}\text { Características } \\
\text { Mecánicas }\end{array}$} \\
\hline & $\begin{array}{c}\mathbf{C} \\
\text { Máx. }\end{array}$ & $\begin{array}{l}\text { Mn } \\
\text { Máx. }\end{array}$ & $\begin{array}{c}\mathbf{P} \\
\text { Máx. }\end{array}$ & $\begin{array}{c}\mathbf{S} \\
\text { Máx. }\end{array}$ & $\begin{array}{c}\text { Cr } \\
\text { Máx. }\end{array}$ & \begin{tabular}{|c|} 
Cu \\
Máx.
\end{tabular} & $\begin{array}{c}\text { Ni } \\
\text { Máx. }\end{array}$ & $\begin{array}{c}\text { Mo } \\
\text { Máx. }\end{array}$ & $\begin{array}{c}\mathbf{V} \\
\text { Máx. }\end{array}$ & $\begin{array}{c}\text { Resistencia a } \\
\text { la tracción } \\
\text { (mínima) }\end{array}$ & $\begin{array}{l}\text { Fluencia } \\
\text { (mínima) }\end{array}$ & $\begin{array}{l}\text { Alargamiento } \\
\text { (mínimo) }\end{array}$ \\
\hline & $\%$ & $\%$ & $\%$ & $\%$ & $\%$ & $\%$ & $\%$ & $\%$ & $\%$ & $\mathrm{Kg} / \mathrm{mm}^{2}$ & $\mathrm{Kg} / \mathrm{mm}^{2}$ & $\%$ \\
\hline B & 0,35 & 1,2 & 0,05 & 0,045 & 0,40 & 0,40 & 0,40 & 0,40 & $\mathbf{0 , 0 8}$ & 42 & 25 & 30 \\
\hline
\end{tabular}

Tabla 1.

\section{Probetas de radiación solar y oxidación para el receptor}

1. Probeta 1_radiacion solar. Tubo de acero SAE 1020, estructural con costura, sin prueba hidráulica, de diámetro exterior de 2" por espesor $2 \mathrm{~mm}$. Longitud de probeta $500 \mathrm{~mm}$. Con cupla de $3 / 4$ de pulgada en sus extremos para alojar termocupla y válvula de seguridad. En el interior de la probeta se introduce el fluido térmico (aire o agua destilada). (Groover, 2007). Figura 5.

2. Probeta 2_radiacion solar. Tubo de acero ASTM A53, grado B, de conducción con costura, con prueba hidráulica, de diámetro exterior de 60,3 por espesor $3,9 \mathrm{~mm}$. Ancho de probeta $500 \mathrm{~mm}$. Con cupla de $3 / 4$ de pulgada en sus extremos para alojar termocupla y válvula de seguridad. En el interior de la probeta se introduce el fluido térmico (aire o agua destilada). (Groover, 2007). Figura 5.

3. Probeta 1_oxidación. Tubo de acero SAE 1020, estructural con costura, sin prueba hidráulica, de diámetro exterior de 2" por espesor $2 \mathrm{~mm}$. Ancho de probeta 20mm. (Groover, 2007). Figura 6.

4. Probeta 2_oxidación. Tubo de acero ASTM A53, grado B, de conducción con costura, con prueba hidráulica, de diámetro exterior de 60,3 por espesor 3,9 mm. Ancho de probeta 20mm. (Groover, 2007). Figura 6.

\section{Ensayo de oxidación}

Para la realización de ensayos de oxidación a intemperie se eligió un lugar con extrema humedad. Tiempo de prueba 60 días. Se puede observar en la figura 7, que la probeta 1 se oxido más que la probeta 2. Esto se debe por su diferencia de composición química. El tiempo de ensayo a intemperie fue de 90 días con temperaturas entre 10 y $35^{\circ} \mathrm{C}$ en La Plata, Provincia de Buenos Aires. (García-Segura, Fernández-García, Ariza, Sutter, \& Valenzuela, 2016).

El material seleccionado no soporta condiciones externas como la humedad ambiente. Esto genera corrosión en este tipo de acero el cual se ve obligado a una protección que además sirva de superficie absorbedora de radiación solar. (García-Segura, FernándezGarcía, Ariza, Sutter, \& Valenzuela, 2016); (Groover, 2007). 


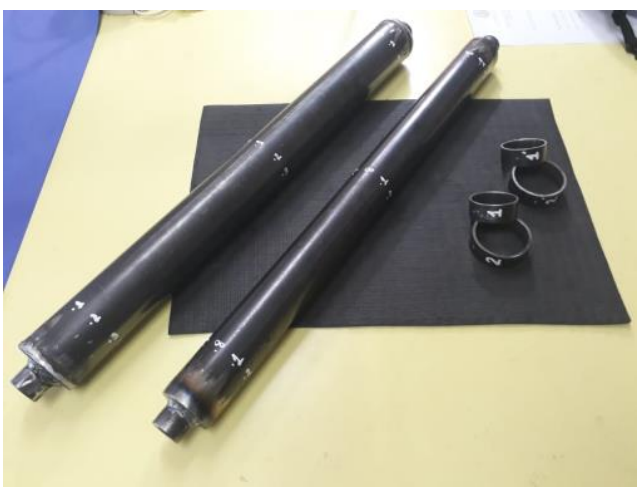

Figura 5, probetas para radiación solar.

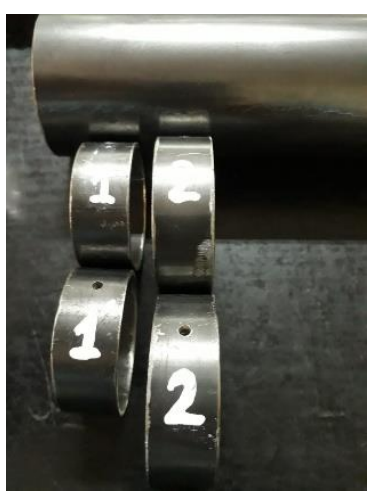

Figura 6

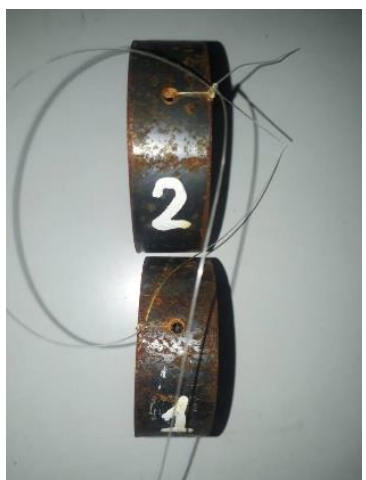

Figura 7

\section{Ensayo de radiación solar}

Para la realización de ensayos de radiación se realizó un protector de vidrio de sección hexagonal con facetado vítreo para evitar pérdidas de temperatura y mejorar la eficiencia térmica en el receptor. Se utilizó un prototipo para la ubicación de las probetas 1 y 2 receptor, con una distancia focal entre 900 y $1000 \mathrm{~mm}$. Entre el receptor y 1/16 (un dieciseisavo) de concentrador curvado reflectivo, de $50 \mathrm{~cm}$ de ancho por $84 \mathrm{~cm}$ de longitud y $4 \mathrm{~mm}$ de espesor, con curva parabólica de flecha entre 40 y $27 \mathrm{mmcon}$ espejos curvados, (Fuqiang et al., 2017). Figuras 8, 9 y 10.

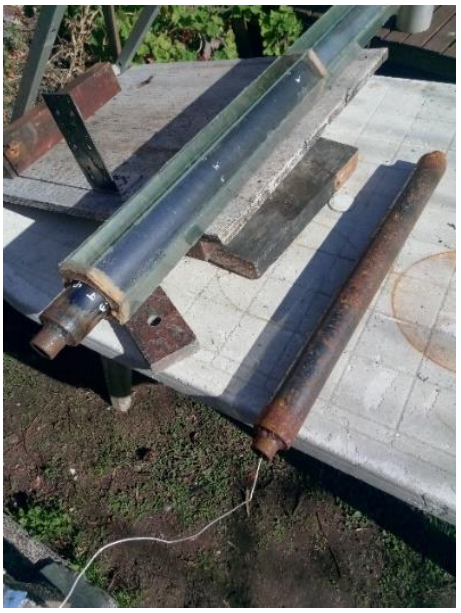

Figura 8, receptor testigo

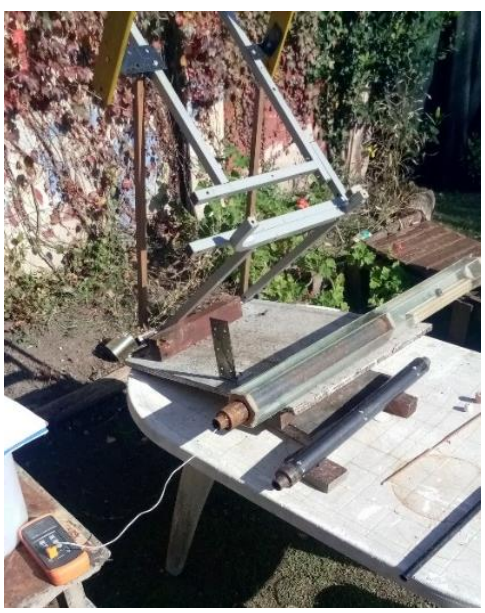

Figura 9, receptor hexágono

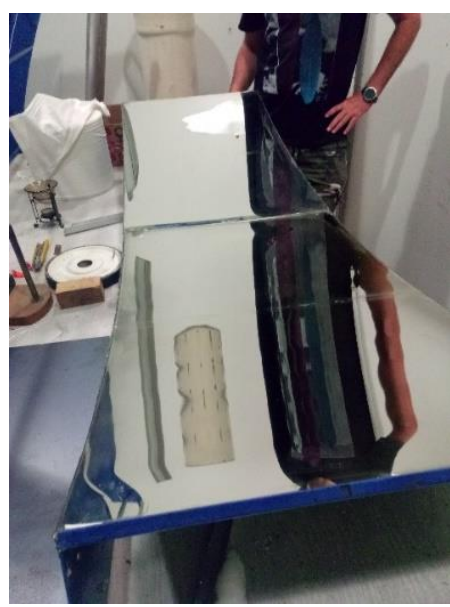

Figura 10, concentrador

Ensayos realizados el día 28 de mayo de 2020 con radiación medida directa (sistema ecuatorial). Tabla 2. Desde las 11:00 horas, hasta las 13:30 horas se realizó el estudio directo del sistema de Hexágono óptico segmentado.

\begin{tabular}{|c|c|c|c|c|}
\hline Horario & $\begin{array}{l}\text { Receptor testigo } \\
\text { Temperatura }{ }^{\circ} \mathrm{C}\end{array}$ & $\begin{array}{l}\text { Ambiente } \\
\text { Temperatura }{ }^{\circ} \mathrm{C}\end{array}$ & $\begin{array}{l}\text { Receptor dentro del } \\
\text { Hexágono. } \\
\text { Temperatura }{ }^{\circ} \mathbf{C}\end{array}$ & $\begin{array}{l}\text { Radiación } \\
\text { aproximada W/m } 2\end{array}$ \\
\hline $11: 10$ & 08 & 13 & 15 & 850 \\
\hline $11: 30$ & 13 & 15 & 50 & 850 \\
\hline $11: 40$ & 16 & 16 & 58 & 890 \\
\hline 11:44 & 22 & 18 & 57 & 890 \\
\hline $11: 56$ & 32 & 19 & 60 & 900 \\
\hline 12:04 & 36 & 20 & 62 & 950 \\
\hline $12: 10$ & 60 & 19 & 37 & 950 \\
\hline $12: 40$ & 48 & 19 & 57 & 900 \\
\hline 13:00 & 34 & 20 & 59 & 900 \\
\hline $13: 15$ & 32 & 19 & 61 & 860 \\
\hline
\end{tabular}

Tabla 2. Cambio de receptor testigo por receptor dentro del hexágono 


\section{Conclusiones}

La eficiencia del sistema de absorbedor por donde circula un fluido en condiciones de temperaturas adecuadas, $\left(100\right.$ a $\left.300^{\circ}\right)$, será de alta eficiencia dependiendo del fluido térmico y de los materiales compuestos del absorbedor, aleaciones de aluminio, aleaciones de acero de alta presión, aleaciones de cobre, además de las terminaciones superficiales que resuelvan problemas de corrosión y mejoren la absorción de radiación solar, (Hussein, 2016); (Groover, 2007).

Si el absorbedor está encerrado o encapsulado sobre una cobertura de vidrio al vacío la eficiencia en más del $97 \%$ En nuestro caso el sistema de absorbedor trabajara en dos modos, libre y compuesto con un sistema vítreo de protección. Esto asegurará en los sistemas fijos, la amplitud de energía útil al proteger el sistema de las perdidas por convecciones. (Fuqiang et al., 2017); (Groover, 2007).

Las aplicaciones en el campo de la generación de calor de proceso son muy grandes para la Industria que necesite entre 100 y 300 grados centígrados, secaderos agro-tecnológicos, industrias metalmecánicas, Residuos sólidos urbanos (RSU) Utilizando sistemas de CCP fijos y heliostatos en movimiento, (Hussein, 2016)

Desde el año 2013 el LOCE-FCAG-UNLP junto con la UTN-FRLP está desarrollando proyectos de investigación y transferencia al medio productivo basados en la ESTC o Heliotermia Estos trabajos, (Matos, 2017); (L. Martorelli et al., 2020); (L. C. Martorelli, n.d.); (Mujica et al., 2019); (Parabólico, n.d.); (Panaroni et al., 2017), nos han permitido evaluar que este tipo de energía sustentable, constituyen un nuevo y moderno campo de desarrollo tecnológico para el País, abriendo nuevas áreas de gestión industrial, recursos humanos, ingeniería de diseño, macroeconomías regionales, gestionabilidad, durabilidad y nula contaminación ambiental al cabo de su ciclo de vida.

\section{Referencias}

Fernández-García, A., Zarza, E., Valenzuela, L., \& Pérez, M. (2010). Parabolic-trough solar collectors and their applications. Renewable and Sustainable Energy Reviews, 14(7), 1695-1721. https://doi.org/10.1016/J.RSER.2010.03.012

Fuqiang, W., Ziming, C., Jianyu, T., Yuan, Y., Yong, S., \& Linhua, L. (2017, November 1). Progress in concentrated solar power technology with parabolic trough collector system: A comprehensive review. Renewable and Sustainable Energy Reviews. Pergamon. https://doi.org/10.1016/j.rser.2017.05.174

García-Segura, A., Fernández-García, A., Ariza, M. J., Sutter, F., \& Valenzuela, L. (2016). Durability studies of solar reflectors: A review. Renewable and Sustainable Energy Reviews, 62, 453-467. https://doi.org/10.1016/J.RSER.2016.04.060

Gordon, J. (2013). Solar energy: the state of the art. Retrieved from https://books.google.com.ar/books?hl=es\&lr=\&id=RdeNAQAAQBAJ\&oi=fnd\&pg=PP1\&dq=Gordon,+J.M.:+Solar+Energy:+T he+State+of+the+Art.+Taylor+\%26+Francis,+2013,+ISBN+9781134275823.+https://books.google.com.ar/books\%3F+id\%3DR deNAQAAQBAJ.\&ots=y_cHPIoTwt\&sig=Lq3cR3ztmyHummPrhn9t7o0F16s

Groover, M. P. (2007). Fundamentos de manufactura moderna. Gestión de procesos.

Hussein, A. K. (2016). Applications of nanotechnology to improve the performance of solar collectors - Recent advances and overview. Renewable and Sustainable Energy Reviews, 62, 767-792. https://doi.org/10.1016/j.rser.2016.04.050

Luján, G. G.-, Aires, B., \& 2004, undefined. (n.d.). Notas sobre radiación solar. Researchgate.Net. Retrieved from https://www.researchgate.net/profile/Hugo_Grossi_Gallegos/publication/311375862_Notes_on_Solar_Radiation_en_espanol/lin ks/584338c208ae2d2175637d92/Notes-on-Solar-Radiation-en-espanol.pdf

Martorelli, L. C. (n.d.). “ $3^{\circ}$ Foro Patagónico de Energías Sustentables ” Diseño de sistemas ópticos y análisis de errores en focos lineales para concentración solar, (1).

Martorelli, L., Panaroni, D., Alvarez, C., Corujo, L., \& Cervini, F. (2020). Energía solar térmica de concentración , experimentación de prototipos para plantas solares, 13-15.

Matos, A. (2017). Xlii jornadas iram universidades, 2017.

Mujica, M., Panaroni, D., \& Corujo, L. (2019). Análisis de un foco lineal en un Colector Cilindro Parabólico para.

Panaroni, D., Mujica, M., Matos, M. A., Corujo, L., Luna, A. C., \& Martorelli, L. (2017). Diseño óptico de un colector cilíndrico parabólico inclinado para generación directa de vapor. Retrieved from https://digital.cic.gba.gob.ar/handle/11746/6785

Parabólico, C. (n.d.). Formato e Instrucciones para la preparación de los trabajos a presentar en el " $3^{\circ}$ Foro Patagónico de Energías Sustentables " Caracterización del error de pendiente o Slope en un Colector, (1), 1-6. 